\title{
Normalbefunde nach aufwendiger Diagnostik
}

\section{Diagnostische Untersuchungen bei Symptomen mit geringer Wahr- scheinlichkeit auf ernsthafte Erkran- kungen sind kostenträchtig und füh- ren in der Regel zu Normalbefunden. Das beruhigt die Ärzte, nicht aber die Patienten.}

- Die Autoren suchten nach randomisierten, kontrollierten Studien, in denen bei Symptomen wie Dyspepsie, Rückenschmerzen, Palpitationen, akute und chronische Kopfschmerzen diverse diagnostische Maßnahmen, vor allem Endoskopien und bildgebende Verfahren nach Zufallskriterien entweder eingesetzt oder unterlassen wurden. Gleichzeitig mussten Merkmale wie Beruhigung, Angst, Lebensqualität und Zufriedenheit der Patienten kurz- und langfristig (weniger bzw. länger als drei Monate) nach der Diagnostik eruiert werden. Es gab 14 Studien mit 3828 Patienten.

Eine positive Folge der Diagnostik war eine geringe, nicht signifikante Ab- nahme nachfolgender Kontrolluntersuchungen. Abhängig vom Ausgangssymptom mussten allerdings 16 bis 26 Untersuchungen durchgeführt werden, damit eine Nachfolgeuntersuchung unterblieb. Auf die Befindlichkeiten der Patienten wie z.B. die Sorgen über eine evtl. Erkrankung, unspezifische Ängste und die Ausgangsbeschwerden hatten Normal-

\section{Kommentar}

Diagnostische Untersuchungen werden häufig bei unspezifischen Beschwerden angeordnet, um ernsthafte Erkrankungen trotz geringer Vortestwahrscheinlichkeit auszuschließen. Doch derartige Untersuchungen, die überwiegend keinen krankhaften Befund aufdecken, und die Mitteilung an den $\mathrm{Pa}$ tienten „Alle ihre Untersuchungen waren normal" können die Patienten weder beruhigen noch langfristig von ihren Beschwerden befreien. Zudem hat Diagnostik bei niedriger Vortestwahrscheinlichkeit auch ernsthafte Nebenwirkungen wie falsch positive Befunde, die zu Traumatisierung der Patienten befunde nach der Diagnostik keinen Einfluss. Andererseits führte die Diagnostik in Abhängigkeit vom Ausgangsymptom zu 0,5 bis $3 \%$ ernsthaften Diagnosen.

\section{- A. Rolfe und C. Burton \\ Reassurance after diagnostic testing with a low pretest probability of serious disease. JAMA Intern. Med. 2013; 173: 407-416}

und kostenträchtiger Anschlussdiagnostik mit weiteren Nebenwirkungen führen. In der vorliegenden Untersuchung wurden 0,5 bis 3\% ernsthafte Erkrankungen aufgedeckt. Unter der Annahme, dass die diagnostischen Methoden eine Sensitivität und Spezifität von $90 \%$ erreichen, kommen auf jedes richtig positive Resultat 4 bis 16 falsch positive Ergebnisse. Resümee der Autoren: Aufwendige Untersuchungen bei den genannten Beschwerden lohnen nicht, weil sie weder die Patienten beruhigen noch im Sinne von Vorsorgeuntersuchungen ergiebig sind.

H. HOLZGREVE =

\section{Rotes Melanom}

Eine 48-jährige Frau hatte seit zwei Jahren am linken Oberarm einen roten, nicht druckschmerzhaften runden Knoten bemerkt, der bei 2 Uhr eine blaugraue Pigmentierung aufwies (Abb. A). Bei der Dermatoskopie erkannte man eine unregelmäBige, rötliche diffuse Pigmentierung und
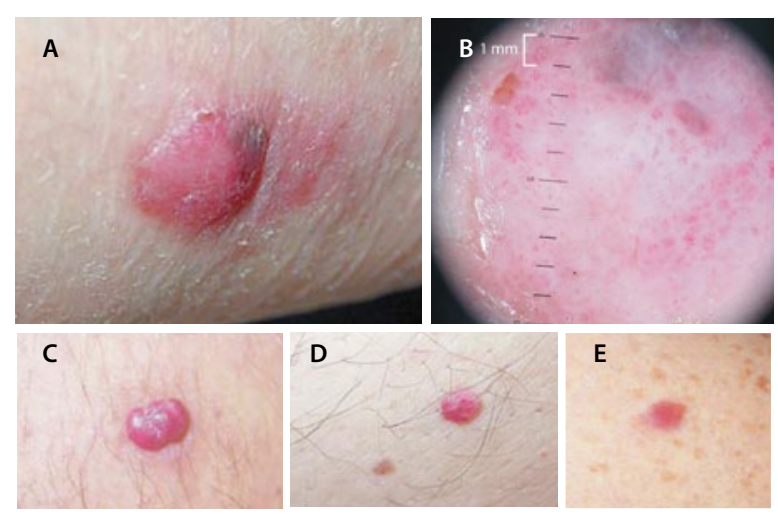

E

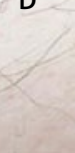

Amelanotische Melanome (A, B) können leicht mit pyogenen Granulomen (C), Angiomen (D) oder dermalen Naevi (E) verwechselt werden. ein diffuses polymorphes Gefäßmuster (Abb. B). Die farblich unterschiedliche Region wies unregelmäBig stehende blaugraue Knötchen auf. Bei der histologischen Untersuchung bestätigte sich der klinische und dermatoskopische Verdacht eines amelanotischen
Melanoms mit einer Breslow-Dicke von 3,9 $\mathrm{mm}$. Im weiteren Verlauf entdeckte man auch einen Sentinel-Lymphknoten, in dem sich Zellen eines metastasierten Melanoms fanden. Man führte eine axilläre Lymphadenektomie durch. Bei der vollständigen Diagnostik ergaben sich Hinweise auf zerebrale und Skelettmetastasen, sodass die Patienten auch eine adjuvante Chemotherapie erhielt.

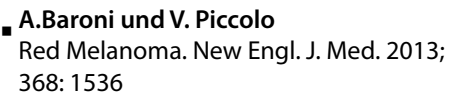

- A.Baroni und V. Piccolo

Red Melanoma. New Engl. J. Med. 2013; 368: 1536

\section{Kommentar}

Amelanotische Melanome können vielen benignen Hauterkrankungen ähneln, zum Beispiel einem pyogenen Granulom (Abb. C), einem Angiom (Abb.D) oder einem dermalen Naevus (Abb. E). Sie werden daher nicht selten fälschlicherweise mit Diathermie oder dem Laser behandelt. Der Fall zeigt die Notwendigkeit einer sorgfältigen und kenntnisreichen Untersuchung von roten Knötchen durch einen Fachmann auf. Mit einer histologischen Klärung sollte man nicht zu zögerlich sein. H. S. FüESSL = 